\title{
Singular Value Decomposition Based Features for Automatic Tumor Detection in Wireless Capsule Endoscopy Images
}

\author{
Vahid Faghih Dinevari, Ghader Karimian Khosroshahi, and Mina Zolfy Lighvan \\ Electrical and Computer Engineering Department, University of Tabriz, Tabriz 51666 16471, Iran \\ Correspondence should be addressed to Vahid Faghih Dinevari; v.faghih@tabrizu.ac.ir
}

Received 11 January 2016; Accepted 11 April 2016

Academic Editor: Alberto Borboni

Copyright ( 2016 Vahid Faghih Dinevari et al. This is an open access article distributed under the Creative Commons Attribution License, which permits unrestricted use, distribution, and reproduction in any medium, provided the original work is properly cited.

\begin{abstract}
Wireless capsule endoscopy (WCE) is a new noninvasive instrument which allows direct observation of the gastrointestinal tract to diagnose its relative diseases. Because of the large number of images obtained from the capsule endoscopy per patient, doctors need too much time to investigate all of them. So, it would be worthwhile to design a system for detecting diseases automatically. In this paper, a new method is presented for automatic detection of tumors in the WCE images. This method will utilize the advantages of the discrete wavelet transform (DWT) and singular value decomposition (SVD) algorithms to extract features from different color channels of the WCE images. Therefore, the extracted features are invariant to rotation and can describe multiresolution characteristics of the WCE images. In order to classify the WCE images, the support vector machine (SVM) method is applied to a data set which includes 400 normal and 400 tumor WCE images. The experimental results show proper performance of the proposed algorithm for detection and isolation of the tumor images which, in the best way, shows $94 \%, 93 \%$, and $93.5 \%$ of sensitivity, specificity, and accuracy in the RGB color space, respectively.
\end{abstract}

\section{Introduction}

Gastrointestinal (GI) tract diseases are so common all over the world. Therefore, the possibility of direct observation of the GI tract is beneficial for treatment of these patients. None of the traditional methods like endoscopy and colonoscopy can reach the small intestine which is the mean body of the GI tract. This limitation occurs due to the complex structure of the small intestine. Wireless capsule endoscopy is a new instrument which helps doctors achieve this purpose and alleviates the pain caused by the traditional endoscopy for the patients. As a result, it is widely used in hospitals. WCE video data identification is done by physicians, which takes a long time due to the large number of images. The average number of WCE images in each experiment is about 50,000 which takes around two hours to be reviewed and analyzed $[1,2]$. Furthermore, some abnormalities appear just in one or two frames of the video which might be missed by physicians. On the other hand, variation of abnormalities in size, color, and distribution makes their detection too difficult by naked eyes. All these problems motivate researchers to develop other reliable systems to reduce the great burden of physicians. However, it should be considered that this goal is very challenging because the appearance of varied diseases is so different and the same diseases have lots of variations in color, shape, and size $[3,4]$.

Tumor is one of the GI tract diseases which greatly threaten human health [5]. In case of early detection, this abnormality can be prevented and cured easily; therefore, many studies have been done to propose a computer aided detection (CAD) system to identify tumors in the WCE images. Karkanis et al. utilized color wavelet covariance features and a linear discriminant analysis classifier to detect tumors in the colonoscopy videos. This feature is based on the covariance of the second-order textural measures of the different color bands [6]. Moreover, Li used the wavelet transform and uniform local binary pattern (ULBP) as the texture features for detection of tumors in the WCE images $[7,8]$. Martins et al. utilized the advantage of high directional sensitivity of the discrete curvelet transform and the Gaussian mixture model as a classifier to detect tumors in the WCE images [9]. 
In this work, a new algorithm is proposed which exploits color based texture features and SVM to detect tumor in the WCE images. The proposed method integrates advantages of DWT and SVD to characterize the normal and tumor WCE images. DWT is used for multiresolution image analysis as a preprocessing step. In the following step, the SVD based features which are invariant against rotation are extracted. Finally, SVM which has a better ability to classify high dimensional feature vectors is used to classify the WCE images. The experimental results from the presented data set show that this new scheme achieves an encouraging performance in tumor detection.

This paper is organized as follows. The new wavelet based SVD color texture feature extraction for the WCE images is presented in Section 2. In Section 3, SVM, which was used to classify our data set, is briefly introduced. Section 4 presents the experimental results in detail, including the detection evaluation. Finally, the conclusion will be given in Section 5 .

\section{Texture Features}

Due to development in the image analysis methods, the normal and abnormal regions in the WCE images can be distinguished properly. In the present work, we attempt to design a CAD system to recognize tumor in the WCE images. Tumor and normal WCE images have different color and texture features as shown in Figures 1 and 2. These different appearances motivate us to select color texture feature as an image analyzer. This texture is reddish compared to the normal ones [10] due to the increment of blood capillary density in the tumor. Also, the inflation and inflammation surface of tumors can isolate them from the normal textures [11]. The most important problems in this identification are the time varying illumination environment and the great variety in tumor appearances which can be observed in Figure 1 [3]. Therefore, the CAD system should be powerful enough to overcome these problems.

To have a local analysis, the input WCE images are divided into nine patches according to Figure 3. Since the size of the input image is $256 \times 256$ pixels, the number of 128 $\times 128$ overlapping patches is set to three in both directions. For better image analysis, DWT is applied to each patch to have multiresolution and zooming ability. In addition, the SVD based features which are invariant to rotation are extracted from the created subpatch. Finally, SVM is used to classify the WCE images because of its better ability to classify high dimensional feature vectors. The methodology of the proposed CAD system is shown in Figure 4.

2.1. Discrete Wavelet Transform. Wavelet transform is a powerful tool for multiresolution image analysis [12]. Also, zooming ability and local characterization of the wavelet transform provide a much efficient analysis of the WCE images which contain different scale information. DWT can decompose an image into different subbands with both time information and frequency information that are implemented with a separable filter-bank [13]. To create decomposed subimages that are shown in Figure 5, an image is convoluted with a low pass filter (L) and a high pass filter (H). The LL subimage,
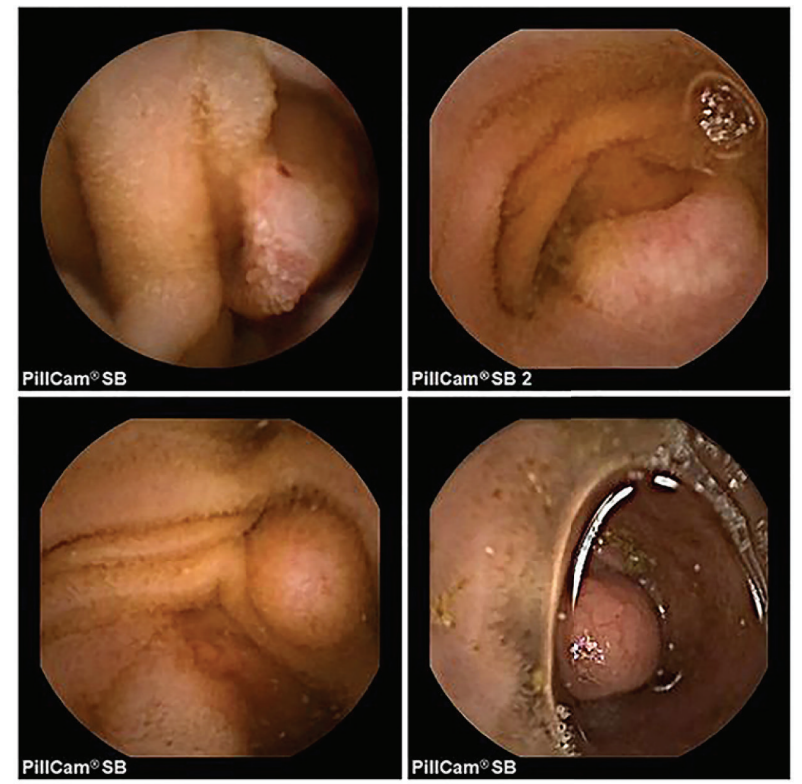

FIGURE 1: Several WCE images with tumor.
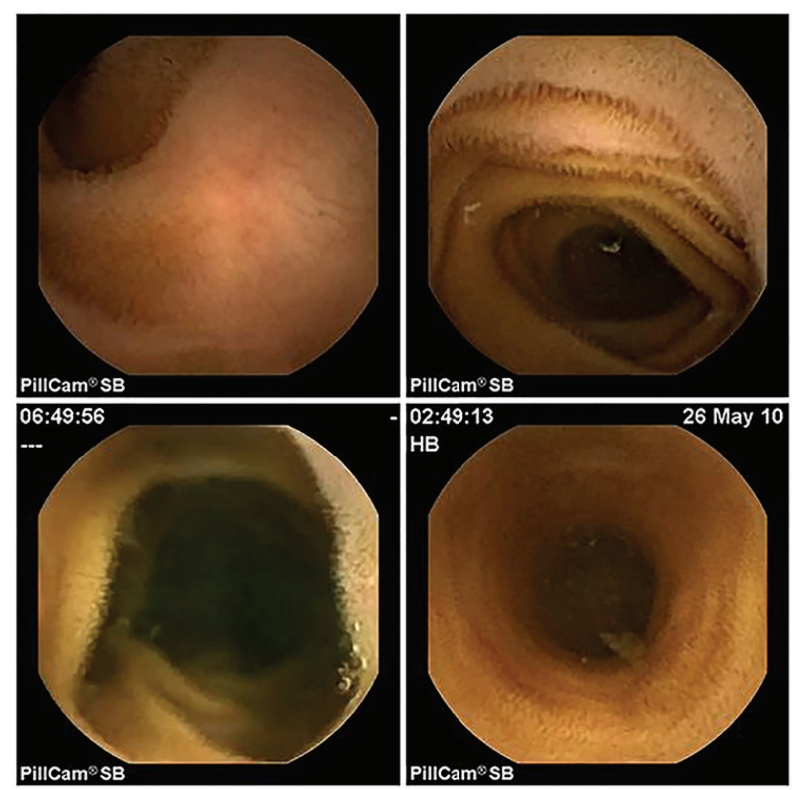

FIgURE 2: Several normal WCE images.

which is called approximation component, is obtained from low pass filtering in both directions. This subimage is very similar to the original image and is used to generate secondlevel decomposition. The other three subimages are called detailed components. HL is derived from low pass filtering along the vertical direction and high pass filtering along the horizontal direction. The remaining $\mathrm{LH}$ and $\mathrm{HH}$ subimages can be explained similarly $[8,14]$. In this study, we only apply the two-level DWT to each color channel of the WCE images. 

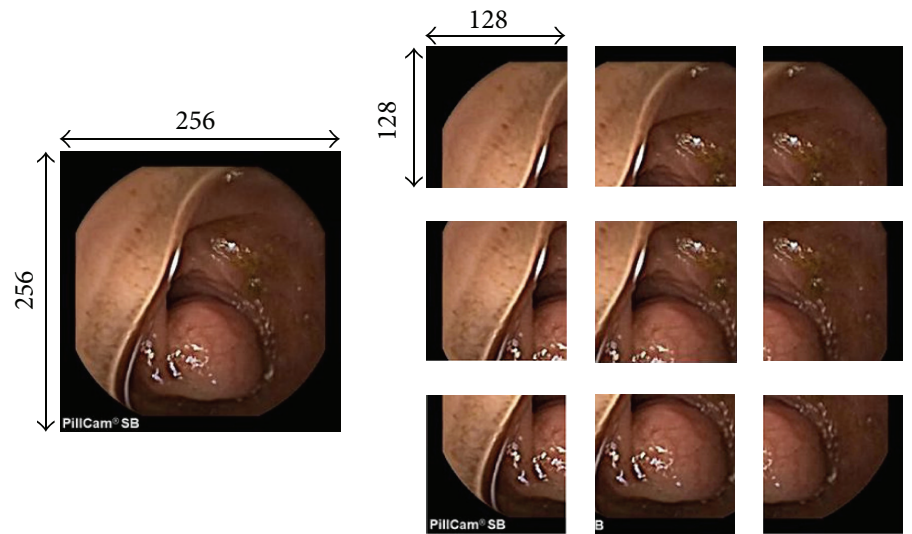

FIgURE 3: Patches chosen from one WCE image.

2.2. Singular Value Decomposition. Singular value decomposition is a tool for exploratory data analysis which dissociates the $m \times n$ data matrix $X$ as follows [15]:

$$
X=\mathrm{USV}^{T} \text {, }
$$

where $U$ is an $m \times m$ orthonormal matrix whose columns are called the left singular vectors or the gene coefficient vectors of $X$. Also, $V$ is an $n \times n$ orthonormal matrix whose columns are called the right singular vectors or the expression level vectors of $X$. Matrix $S$ is an $m \times n$ diagonal matrix which is represented as

$$
S=\left[\begin{array}{ccc}
s_{1} & \cdots & 0 \\
\vdots & \ddots & \vdots \\
0 & \cdots & s_{256}
\end{array}\right]
$$

The diagonal elements of matrix $S$ are called the singular values of $X$ which are used to create the feature vectors [16]. If the input image size is $256 \times 256$, the singular value size will be $1 \times 256$.

In the created subimages, instead of the normal part, there are more texture details in tumors; therefore, the corresponding singular values are bigger than the other parts. This texture difference is used to identify tumors in the WCE images. As mentioned before, textural features are better analyzed in detailed subimages [17], so we use $\mathrm{HL}_{i}, \mathrm{LH}_{i}$, and $\mathrm{HH}_{i}(i=1,2)$ as the bases for the textural feature analysis. Since the WCE images are color ones, each color channel is processed independently. To decrease the feature vector dimension, we compare the accuracy of three color channels in each level of DWT and select the biggest one to extract the SVD based features. Accordingly, the feature vector dimension for each WCE image using the proposed method is $9 \times[(64 \times 3)+(32 \times 3)]=2592$, as illustrated in Figure 6.

In this work, RGB (red, green, and blue) and HSV (hue, saturation, and value) color spaces are used to find the best color space for our feature extraction method. The RGB model is the most convenient color space in image and video processing applications because of the high correlation among the RGB channels [18]. On the other hand, the HSV color model separates color information into chromaticity and intensity, and it is invariant to illumination direction and illumination intensity [19]. Also, the HSV color space is very close to the perceived colors by the human being [6] These properties have made the HSV model less sensitive to illumination variation which is a common problem of the WCE images as the battery weakens over time.

\section{Support Vector Machine}

Support vector machine as an optimal marginal classifier is developed by Vapnik [20]. SVM has been successfully employed in lots of applications providing satisfying results compared to the other methods [7]. The SVM algorithm processes data by minimum classification structural error and good ability to tackle high dimensional problems. As previously mentioned, the SVM classifier, with strong generalization capability, is able to overcome the problem of having great variations in tumor appearances in the WCE images [21].

SVM uses a training set to build a binary classifier which separates the positive and negative samples. The training set is composed of input vectors of $x_{i} \in R^{N}$ and the corresponding target values of $y_{i} \in\{-1,1\}$ for $(i=1 \cdots m)$. The aim is to find a function which correctly classifies the test data as a positive or negative sample.

The SVM algorithm finds a hyperplane with maximized separation margin which is defined by the following equation:

$$
w^{T} f(x)+b=0
$$

where $f(x)$ is a nonlinear mapping from the input space to the bigger dimension feature space which has less overlap between the two classes and $b$ is a scalar which can be estimated from the Karush-Kuhn-Tucker complementary condition. The maximized margin hyperplane is calculated by the following equation [20]:

$$
w=\sum_{i=1}^{m}\left(l_{i} y_{i} f\left(x_{i}\right)\right),
$$


Learning
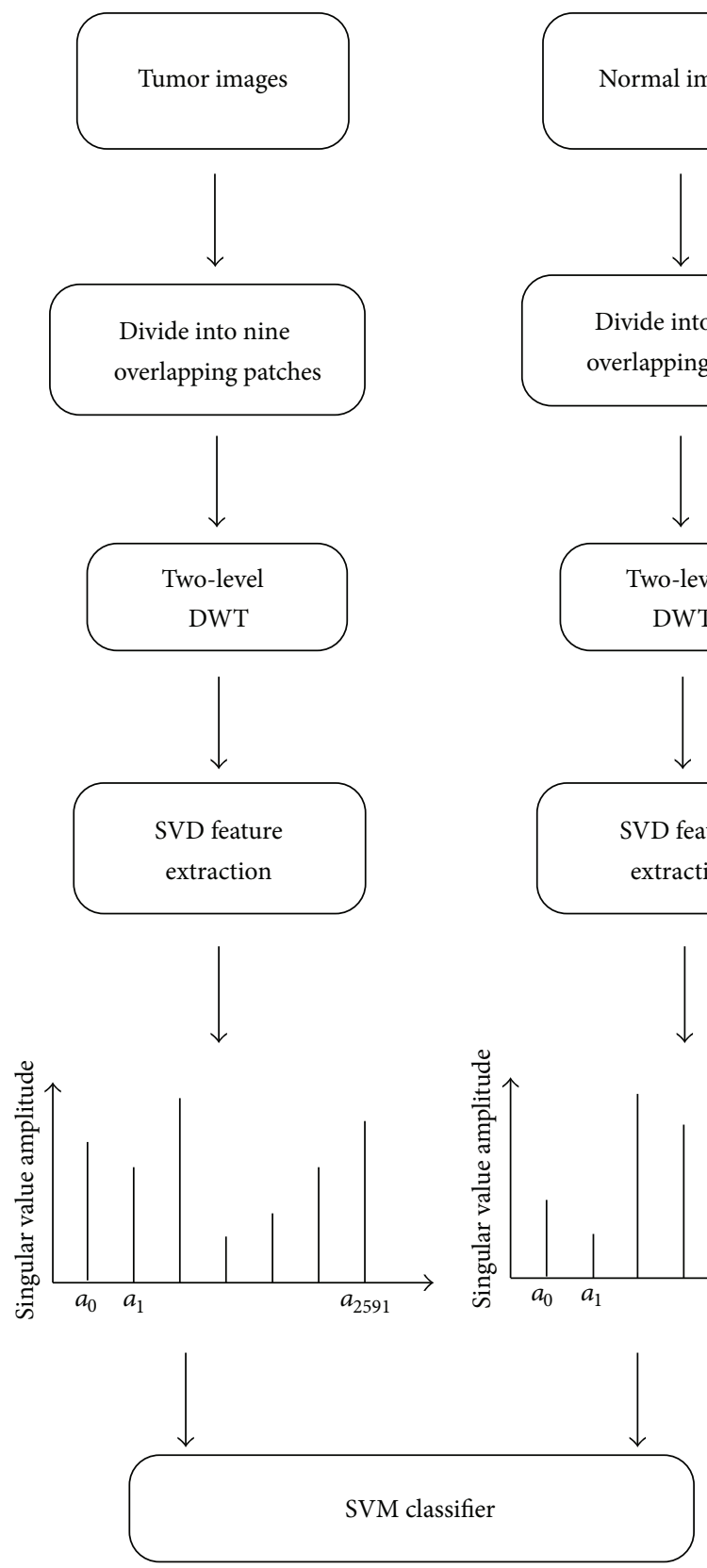

Recognition

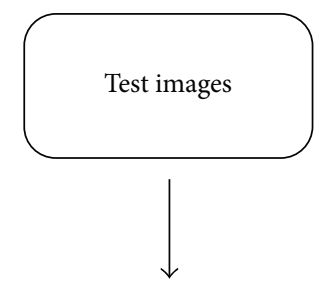

Divide into nine overlapping patches
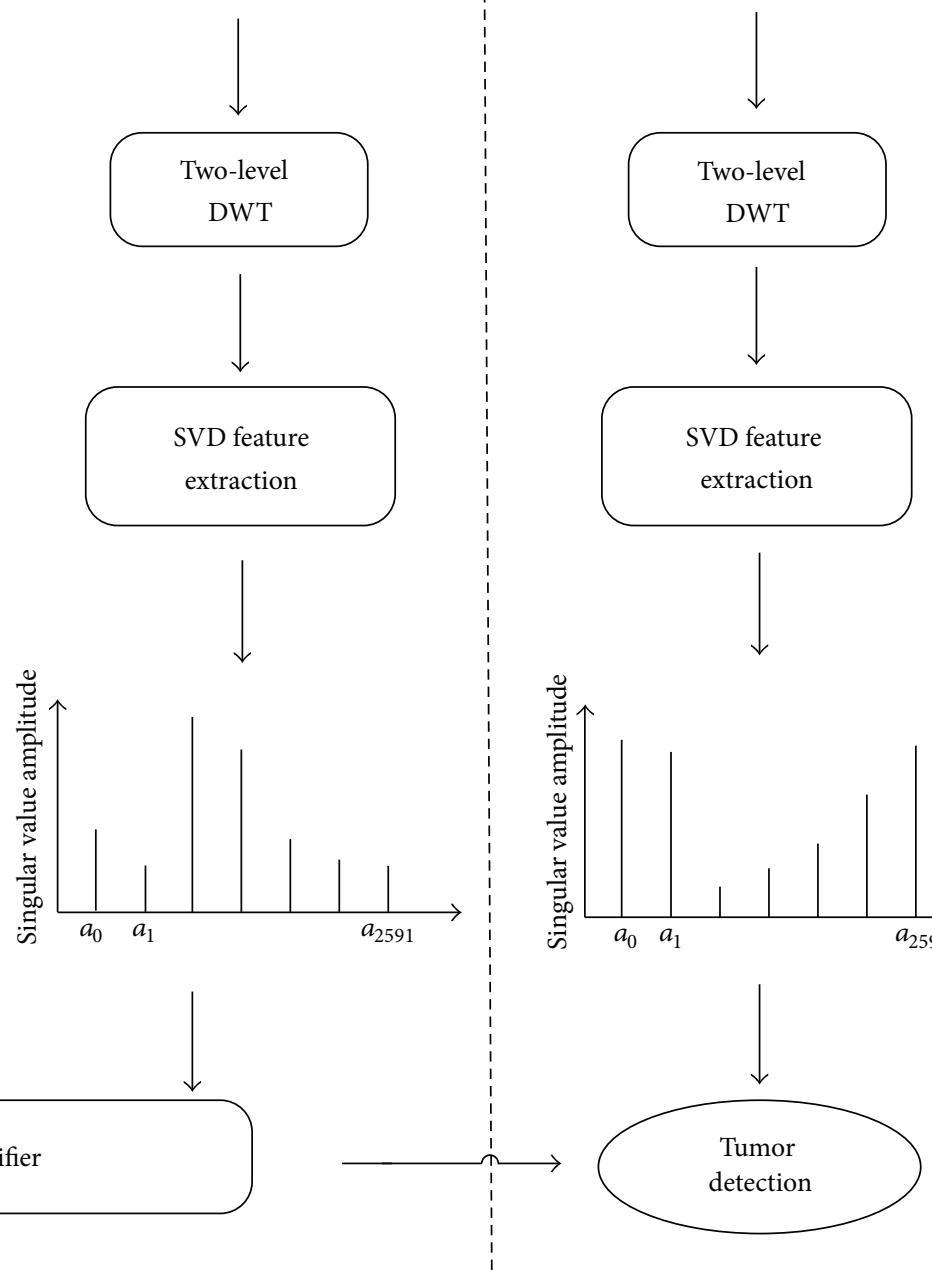

Figure 4: The methodology of the proposed CAD system.

where $l_{i}$ is the Lagrange multiplier that can be calculated by maximization of

$$
\begin{aligned}
L_{D} & =\sum_{i=1}^{m} l_{i}-\frac{1}{2} \sum_{i=1}^{m}\left(\sum_{j=1}^{m} l_{i} l_{j} y_{i} y_{j} K\left(x_{i}, x_{j}\right)\right), \\
K\left(x_{i}, x_{j}\right) & =f^{T}\left(x_{i}\right) f\left(x_{j}\right),
\end{aligned}
$$

where $K$ is a kernel function which is implemented by the linear, polynomial, sigmoid, and radial basis functions (RBF) [22]. The test input vector $x$ is applied to the SVM function and it returns +1 for positive samples and -1 for negative ones with regard to the equation below:

$$
y=\operatorname{sign}\left\{\sum_{i=1}^{m} l_{i} K\left(x_{i}, x\right)+b\right\} .
$$




\section{Results}

For performance evaluation of the proposed algorithm, we use an experimental data set which contains 400 tumor images from 6 pathological cases with different tumor appearances and 400 normal images from 6 normal pathological cases. These images are obtained from PillCam Company (http://www.givenimaging.com/). The resolution of these images is $256 \times 256$ pixels. In addition, to obtain reliable results, some WCE images with a few bubbles and feces, similar to the ones in Figure 7, have been added to our data set.

To extract the performance of the proposed method, each image is manually labeled as a positive sample (tumor image) or a negative one (normal image) to create the ground truth. In this case, 300 normal samples and 300 tumor samples are used as the training set, while the remaining 100 normal samples and 100 tumor ones are used as the test set. This procedure is repeated four times in order to prevent overfitting of the classification results by exchanging the training and testing images. At last, the average recognition rates are used to demonstrate the performance of the proposed method. Success of the WCE image identification is measured due to its sensitivity, specificity, and accuracy which can be explained as follows [6-8]:

\section{Sensitivity}

$$
=\frac{\text { Number of Correct Positive Predictions }}{\text { Number of Positives }},
$$

Specificity

$$
\begin{gathered}
=\frac{\text { Number of Correct Negative Predictions }}{\text { Number of Negatives }}, \\
\text { Accuracy }=\frac{\text { Number of Correct Predictions }}{\text { Number of Positives and negatives }} .
\end{gathered}
$$

Haar wavelet is utilized in this work for implementing DWT because of its superior discriminating power which is illustrated in [23]. Also, to achieve the optimal classification performance, RBF kernel function is used for implementation of SVM.

The performance of the proposed two-level wavelet based SVD features for automatic tumor detection in the RGB color channels is shown in Tables 1, 2, and 3, independently. According to the results, the utmost accuracy is obtained in the first and second levels of DWT from the R and G color channels, respectively, which are used to extract features in the proposed method. The efficiency of the red channel is due to the fact that tumor has more blood vessels compared to the normal textures. Also, the best recognition rate of the proposed method in the first and second levels of DWT in the HSV color channels is obtained from the value channel. These are shown in Tables 4, 5, and 6. Finally, to calculate the performance of the proposed method, the selective color channel is utilized for the first and second levels of DWT to extract the corresponding singular values to create the feature vector. The performance of the proposed algorithm in the RGB and HSV color spaces is shown in Table 7.

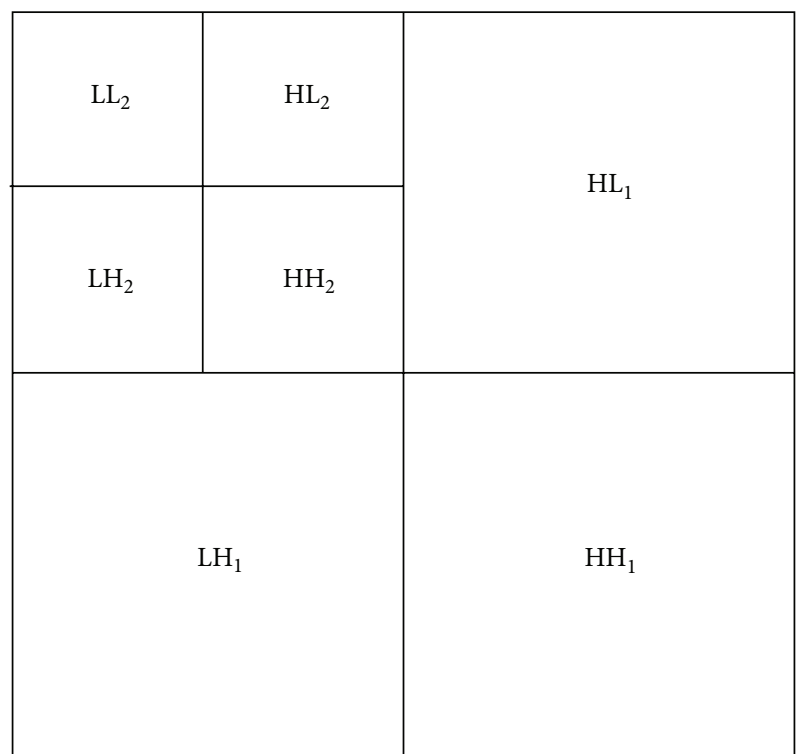

FIgURE 5: Two-level image decomposed using DWT for one color channel.

TABLE 1: Results obtained using the proposed feature in R channel.

\begin{tabular}{lccc}
\hline & Sensitivity & Specificity & Accuracy \\
\hline Level 1 & $93.5 \%$ & $90.5 \%$ & $92 \%$ \\
Level 2 & $90 \%$ & $83 \%$ & $86.5 \%$ \\
Level $1+2$ & $93.75 \%$ & $91.25 \%$ & $92.5 \%$ \\
\hline
\end{tabular}

TABLE 2: Results obtained using the proposed feature in G channel.

\begin{tabular}{lccc}
\hline & Sensitivity & Specificity & Accuracy \\
\hline Level 1 & $90.5 \%$ & $93 \%$ & $91.75 \%$ \\
Level 2 & $86 \%$ & $88 \%$ & $87 \%$ \\
Level $1+2$ & $91 \%$ & $93.5 \%$ & $92.25 \%$ \\
\hline
\end{tabular}

TABLE 3: Results obtained using the proposed feature in B channel.

\begin{tabular}{lccc}
\hline & Sensitivity & Specificity & Accuracy \\
\hline Level 1 & $91 \%$ & $88.5 \%$ & $89.75 \%$ \\
Level 2 & $88 \%$ & $85 \%$ & $86.5 \%$ \\
Level $1+2$ & $93 \%$ & $90 \%$ & $91.5 \%$ \\
\hline
\end{tabular}

To evaluate the performance of the proposed method, it is compared with the wavelet based ULBP which is the latest similar feature proposed by Li and Meng [7] to be used for tumor detection. SVM is used as a classifier for this feature. Tables 8 and 9 show the recognition rates obtained from implanting the wavelet based ULBP in the RGB and HSV color spaces with the current data set.

Finally, the extracted results from the proposed algorithm and two-level wavelet based ULBP in the RGB and HSV color models are compared in Figure 8. The results indicate that the presented SVD based algorithm has a better performance in automatic tumor detection in the WCE images in terms 


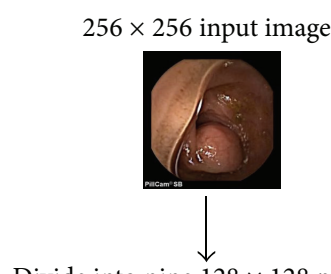

Divide into nine $128 \times 128$ patches
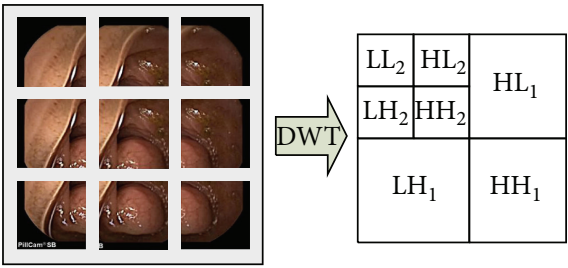
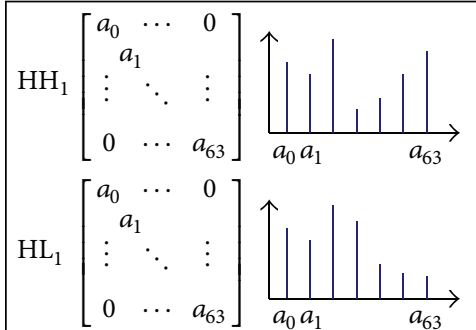

$\mathrm{LH}_{1}\left[\begin{array}{ccc}a_{0} & \cdots & 0 \\ a_{1} & & \\ \vdots & \ddots & \vdots \\ 0 & \cdots & a_{63}\end{array}\right]$
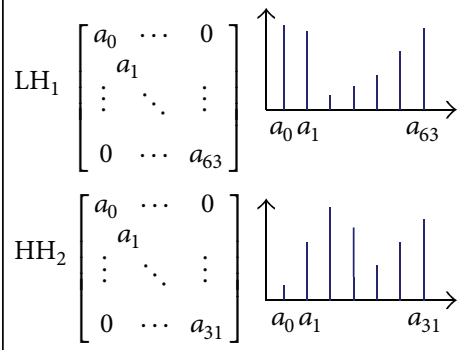

$\mathrm{HL}_{2}$

$\left[\begin{array}{ccc}a_{0} & \cdots & 0 \\ a_{1} & & \\ \vdots & \ddots & \vdots \\ 0 & \cdots & a_{31}\end{array}\right]$
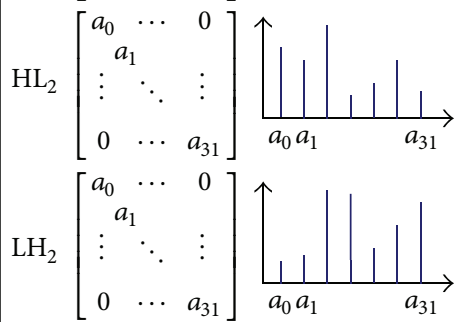

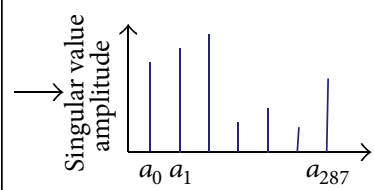

Feature for each $128 \times 128$ patch

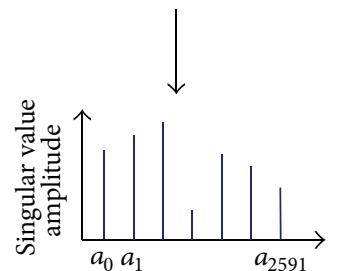

Feature for $256 \times 256$ input image

FIGURE 6: The process for extracting SVD based color texture feature using two-level DWT.
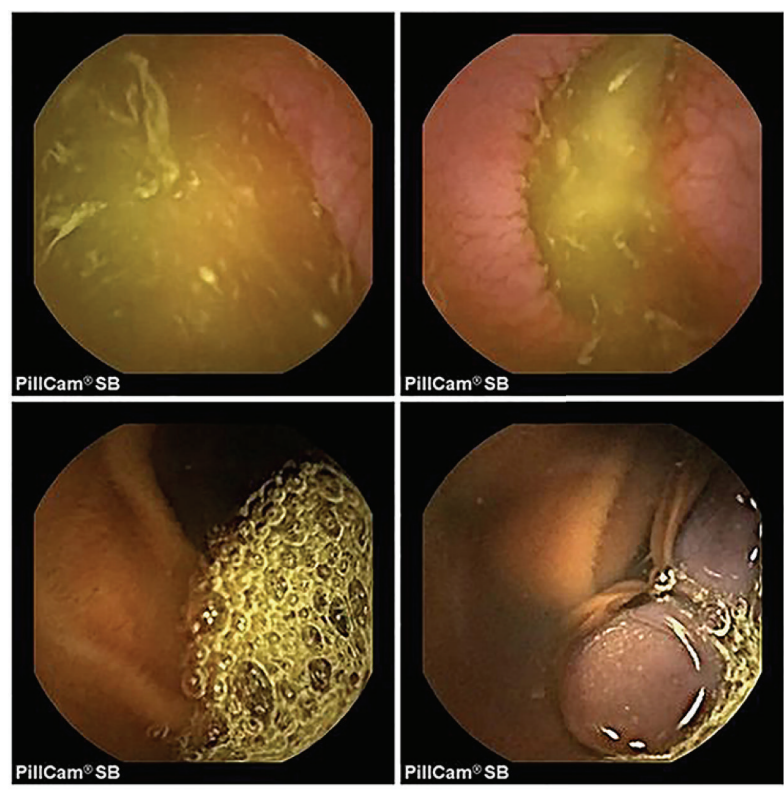

FIGURE 7: Several normal WCE images.

of sensitivity, specificity, and accuracy compared to the twolevel wavelet based ULBP algorithm in both color spaces. The highest accuracy obtained from the proposed algorithm is $93.5 \%$ in the RGB color model while the best recognition rate extracted from employing the two-level DWT + ULBP
TABLE 4: Results obtained using the proposed feature in $\mathrm{H}$ channel.

\begin{tabular}{lccc}
\hline & Sensitivity & Specificity & Accuracy \\
\hline Level 1 & $86.5 \%$ & $80 \%$ & $87.75 \%$ \\
Level 2 & $82.5 \%$ & $81.5 \%$ & $81.5 \%$ \\
Level $1+2$ & $87.5 \%$ & $86.5 \%$ & $89.5 \%$ \\
\hline
\end{tabular}

TABLE 5: Results obtained using the proposed feature in S channel.

\begin{tabular}{lccc}
\hline & Sensitivity & Specificity & Accuracy \\
\hline Level 1 & $94 \%$ & $79 \%$ & $86.5 \%$ \\
Level 2 & $80 \%$ & $83 \%$ & $81.5 \%$ \\
Level 1 + 2 & $93 \%$ & $84 \%$ & $88.5 \%$ \\
\hline
\end{tabular}

TABLE 6: Results obtained using the proposed feature in V channel.

\begin{tabular}{lccc}
\hline & Sensitivity & Specificity & Accuracy \\
\hline Level 1 & $91 \%$ & $90 \%$ & $90.5 \%$ \\
Level 2 & $89.5 \%$ & $85 \%$ & $87.5 \%$ \\
Level $1+2$ & $92.5 \%$ & $90.5 \%$ & $91.5 \%$ \\
\hline
\end{tabular}

is $88.5 \%$ in the same color model. High accuracy obtained by the proposed CAD system demonstrates that the system can overcome the problem of the great variety of tumor appearances in the time varying illumination environments. Better recognition rate of the proposed method for tumor detection in the WCE images is due to the fact that SVD is a better tool to analyze data from different appearance textures. 
TABLE 7: Results obtained using the proposed algorithm in RGB and HSV color spaces.

\begin{tabular}{lcccc}
\hline Color space & $\begin{array}{c}\text { Color channel used for } \\
\text { the first level of the DWT }\end{array}$ & $\begin{array}{c}\text { Color channel used for the } \\
\text { second level of the DWT }\end{array}$ & Sensitivity & Specificity \\
\hline RGB & R channel & G channel & $94 \%$ & $93 \%$ \\
HSV & V channel & V channel & $92.5 \%$ & $93.5 \%$ \\
\hline
\end{tabular}

TABLE 8: Results obtained using DWT + ULBP [7] in RGB color space.

\begin{tabular}{lccc}
\hline & Sensitivity & Specificity & Accuracy \\
\hline Level 1 & $86 \%$ & $85 \%$ & $85.5 \%$ \\
Level 2 & $81 \%$ & $83.5 \%$ & $82.75 \%$ \\
Level $1+2$ & $87 \%$ & $90 \%$ & $88.5 \%$ \\
\hline
\end{tabular}

TABLE 9: Results obtained using DWT + ULBP [7] in HSV color space.

\begin{tabular}{lccc}
\hline & Sensitivity & Specificity & Accuracy \\
\hline Level 1 & $84 \%$ & $86 \%$ & $85 \%$ \\
Level 2 & $79 \%$ & $78 \%$ & $78.5 \%$ \\
Level 1 +2 & $85 \%$ & $90 \%$ & $87.5 \%$ \\
\hline
\end{tabular}

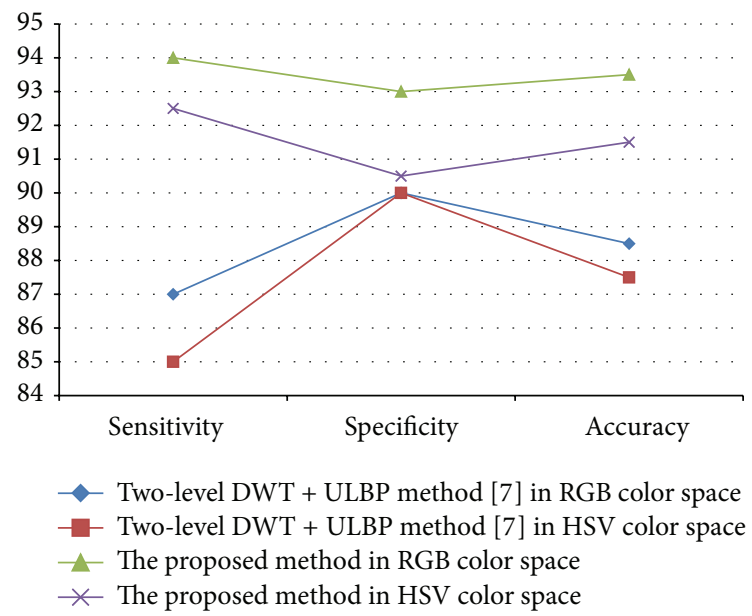

FIGURE 8: Extracted results from the proposed algorithm and twolevel DWT + ULBP method in RGB and HSV color spaces.

On the other hand, local analysis of the WCE images can help in improving the performance of the proposed method.

\section{Conclusions}

A new CAD system has been proposed in this paper for automatic tumor detection in the WCE images. This method locally investigated the input WCE images to extract features. The advantage of the proposed system is based on a new feature extraction method with multiresolution and rotation invariant analysis ability which exploits wavelet based singular value decomposition. In addition, support vector machine with high dimensional classification ability is used to distinguish between the normal and tumor samples in the WCE images. The experimental results from the presented data set which contains 800 WCE images show that this scheme is more powerful in tumor detection. The best recognition rates of $94 \%, 93 \%$, and $93.5 \%$ are achieved in terms of sensitivity, specificity, and accuracy using the twolevel selective wavelet based SVD feature extractor in the RGB color space.

\section{Competing Interests}

The authors declare that they have no competing interests.

\section{References}

[1] D. G. Adler and C. J. Gostout, "Wireless capsule endoscopy," Hospital Physician, vol. 39, pp. 14-22, 2003.

[2] R. Sidhu, D. S. Sanders, K. Kapur, L. Marshall, D. P. Hurlstone, and M. E. McAlindon, "Capsule endoscopy: is there a role for nurses as physician extenders?" Gastroenterology Nursing, vol. 30, no. 1, pp. 45-50, 2007.

[3] J. T. Carlo, D. DeMarco, B. A. Smith et al., "The utility of capsule endoscopy and its role for diagnosing pathology in the gastrointestinal tract," American Journal of Surgery, vol. 190, no. 6, pp. 886-890, 2005.

[4] M. T. Coimbra and J. P. S. Cunha, "MPEG-7 visual descriptorscontributions for automated feature extraction in capsule endoscopy," IEEE Transactions on Circuits and Systems for Video Technology, vol. 16, no. 5, pp. 628-636, 2006.

[5] S. L. Parker, T. Tong, S. Bolden, and P. A. Wingo, "Cancer statistics, 1997," CA: A Cancer Journal for Clinicians, vol. 47, no. 1, pp. 5-27, 1997.

[6] S. A. Karkanis, D. K. Iakovidis, D. E. Maroulis, D. A. Karras, and M. Tzivras, "Computer-aided tumor detection in endoscopic video using color wavelet features," IEEE Transactions on Information Technology in Biomedicine, vol. 7, no. 3, pp. 141-152, 2003.

[7] B. Li and M. Q.-H. Meng, "Tumor recognition in wireless capsule endoscopy images using textural features and SVMbased feature selection," IEEE Transactions on Information Technology in Biomedicine, vol. 16, no. 3, pp. 323-329, 2012.

[8] B. Li, M. Q.-H. Meng, and J. Y. W. Lau, "Computer-aided small bowel tumor detection for capsule endoscopy," Artificial Intelligence in Medicine, vol. 52, no. 1, pp. 11-16, 2011.

[9] M. M. Martins, D. J. Barbosa, J. Ramos, and C. S. Lima, "Small bowel tumors detection in capsule endoscopy by Gaussian modeling of color curvelet covariance coefficients," in Proceedings of the 32nd Annual International Conference of the IEEE Engineering in Medicine and Biology Society (EMBC '10), pp. 5557-5560, September 2010.

[10] I. Flamme, T. Frölich, and W. Risau, "Molecular mechanisms of vasculogenesis and embryonic angiogenesis," Journal of Cellular Physiology, vol. 173, no. 2, pp. 206-210, 1997. 
[11] R. A. Mathias, S. K. Gopal, and R. J. Simpson, "Contribution of cells undergoing epithelial-mesenchymal transition to the tumour microenvironment," Journal of Proteomics, vol. 78, pp. 545-557, 2013.

[12] T. C. Wang and N. B. Karayiannis, "Detection of microcalcifications in digital mammograms using wavelets," IEEE Transactions on Medical Imaging, vol. 17, no. 4, pp. 498-509, 1998.

[13] S. G. Mallat, "Theory for multiresolution signal decomposition: the wavelet representation," IEEE Transactions on Pattern Analysis and Machine Intelligence, vol. 11, no. 7, pp. 674-693, 1989.

[14] W.-L. Lee, Y.-C. Chen, and K.-S. Hsieh, "Ultrasonic liver tissues classification by fractal feature vector based on M-band wavelet transform," IEEE Transactions on Medical Imaging, vol. 22, no. 3, pp. 382-392, 2003.

[15] G. H. Golub and C. F. Van Loan, Matrix Computations, JHU Press, 2012.

[16] B. Nagarajan and V. Devendran, "Singular value decomposition based features for vehicle classification under cluttered background and mild occlusion," Procedia Engineering, vol. 30, pp. 529-534, 2012.

[17] K. W. Abyoto, S. J. Wirdjosoedirdjo, and T. Watanabe, "Unsupervised texture segmentation using multi-resolution analysis for feature extraction," Journal of Tokyo University of Information Sciences, vol. 2, pp. 49-61, 1998.

[18] C. H. Li and P. C. Yuen, "Regularized color clustering in medical image database," IEEE Transactions on Medical Imaging, vol. 19, no. 11, pp. 1150-1155, 2000.

[19] T. Gevers, J. Van de Weijer, and H. Stokman, Color Image Processing: Methods and Applications: Color Feature Detection, CRC Press, New York, NY, USA, 2006.

[20] V. N. Vapnik, The Nature of Statistical Learning Theory, Springer Science \& Business Media, New York, NY, USA, 1995.

[21] L. Wang, Support Vector Machines: Theory and Applications, Springer, Berlin, Germany, 2005.

[22] C. J. C. Burges, "A tutorial on support vector machines for pattern recognition," Data Mining and Knowledge Discovery, vol. 2, no. 2, pp. 121-167, 1998.

[23] L. Semler, L. Dettori, and J. Furst, "Wavelet-based texture classification of tissues in computed tomography," in Proceedings 18th IEEE Symposium on Computer-Based Medical Systems, pp. 265270, Dublin, Ireland, 2005. 


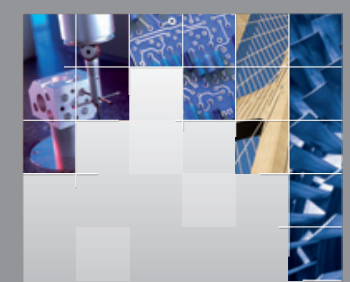

\section{Enfincering}
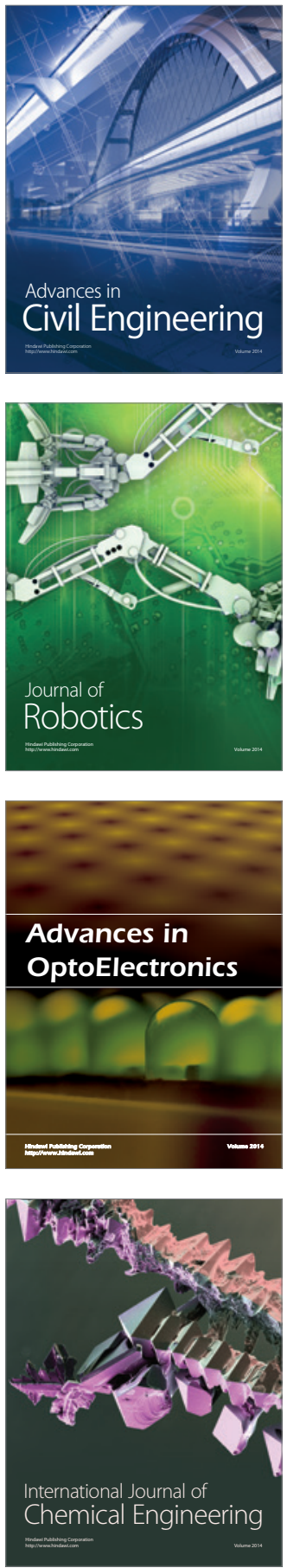

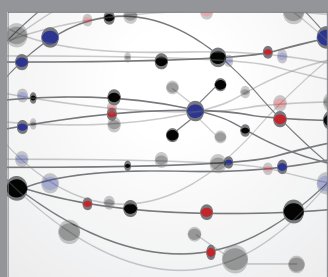

The Scientific World Journal

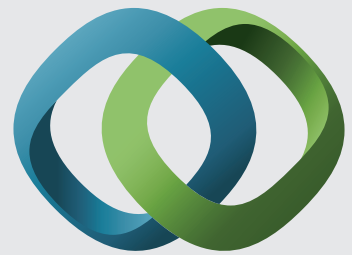

\section{Hindawi}

Submit your manuscripts at

http://www.hindawi.com
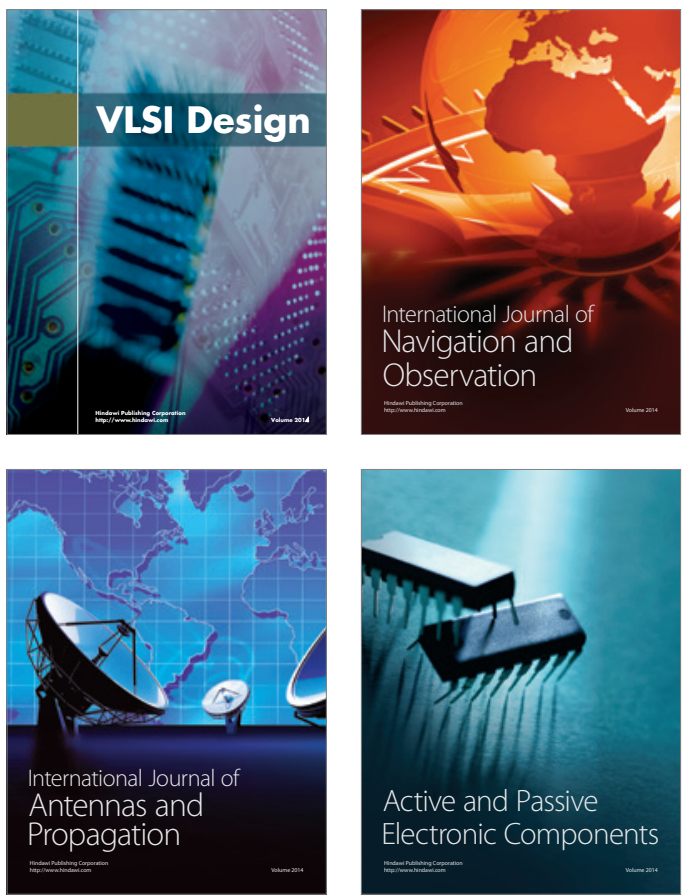
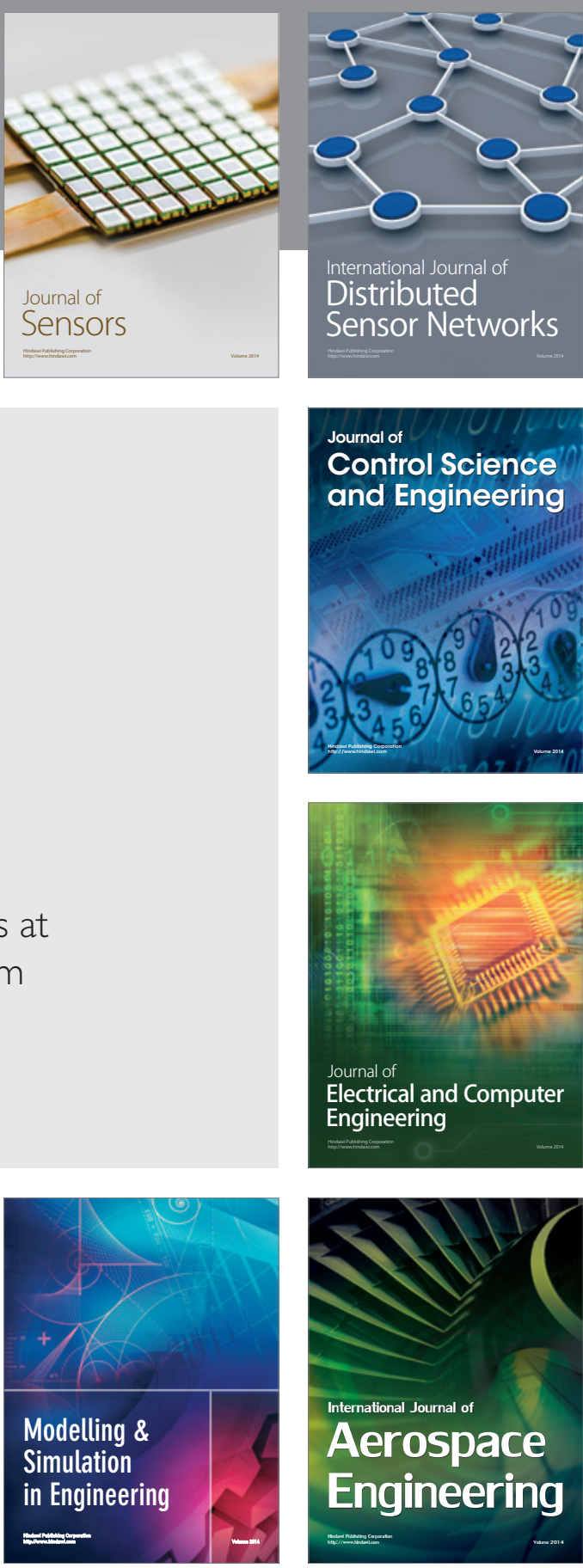

International Journal of

Distributed

Sensor Networks

Journal of

Control Science

and Engineering
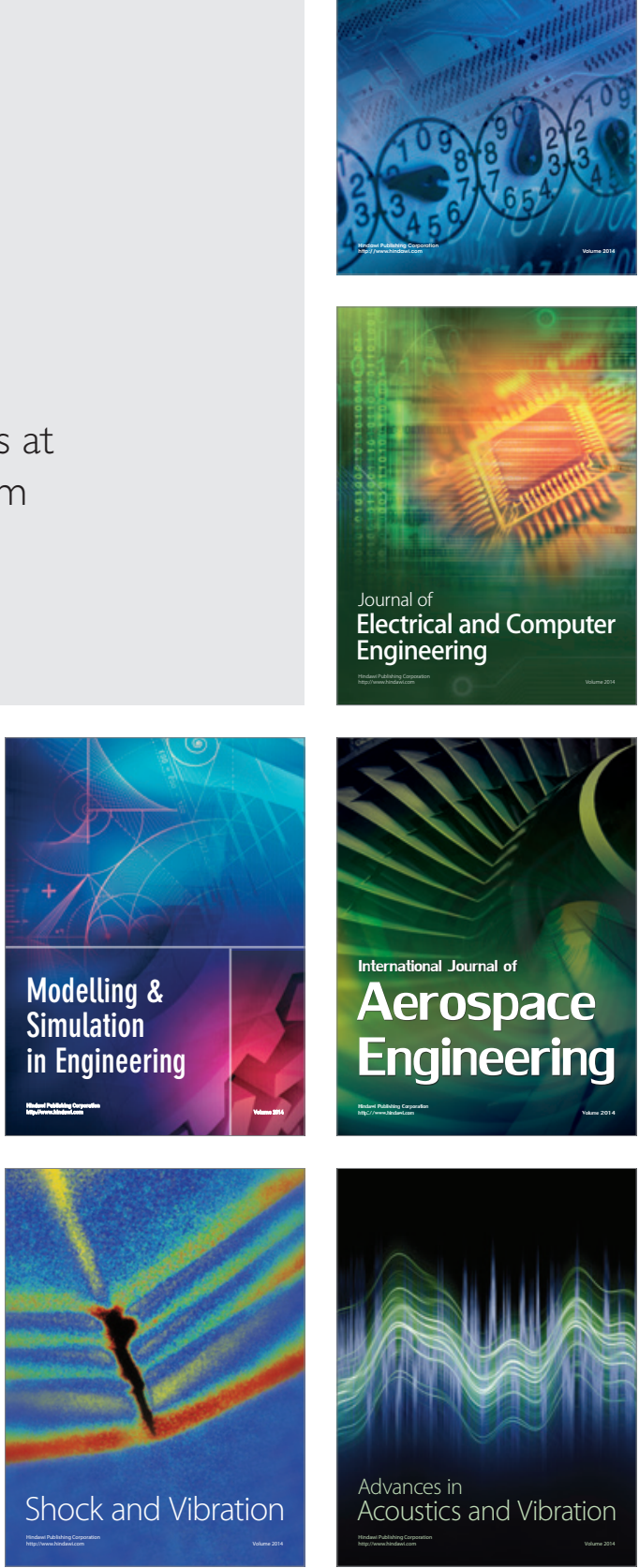